\title{
Nucleic Acid-Based Therapy Approaches for Huntington's Disease
}

\author{
Tatyana Vagner, ${ }^{1}$ Deborah Young, ${ }^{1,2}$ and Alexandre Mouravlev ${ }^{2}$ \\ ${ }^{1}$ Department of Molecular Medicine and Pathology and Centre for Brain Research, The University of Auckland, \\ Auckland 1142, New Zealand \\ ${ }^{2}$ Department of Pharmacology and Clinical Pharmacology and Centre for Brain Research, The University of Auckland, \\ Auckland 1142, New Zealand
}

Correspondence should be addressed to Tatyana Vagner, tatyana.wagner@auckland.ac.nz

Received 8 July 2011; Revised 28 September 2011; Accepted 5 October 2011

Academic Editor: R. M. Damian Holsinger

Copyright (c) 2012 Tatyana Vagner et al. This is an open access article distributed under the Creative Commons Attribution License, which permits unrestricted use, distribution, and reproduction in any medium, provided the original work is properly cited.

\begin{abstract}
Huntington's disease (HD) is caused by a dominant mutation that results in an unstable expansion of a CAG repeat in the huntingtin gene leading to a toxic gain of function in huntingtin protein which causes massive neurodegeneration mainly in the striatum and clinical symptoms associated with the disease. Since the mutation has multiple effects in the cell and the precise mechanism of the disease remains to be elucidated, gene therapy approaches have been developed that intervene in different aspects of the condition. These approaches include increasing expression of growth factors, decreasing levels of mutant huntingtin, and restoring cell metabolism and transcriptional balance. The aim of this paper is to outline the nucleic acid-based therapeutic strategies that have been tested to date.
\end{abstract}

\section{Introduction}

Huntington's disease (HD is an inherited autosomal-dominant disorder characterised by loss of motor control, cognitive decline, psychiatric disturbances, and dementia, which progresses towards death within approximately 20 years of disease onset [1]. It is caused by an expansion of a CAG repeat in the huntingtin gene $(\mathrm{Htt})$ that results in synthesis of an aberrant polyglutamine tract in huntingtin protein (HTT) and leads to neuronal dysfunction and neurodegeneration [2]. GABAergic medium-sized spiny neurons in the striatum are found to be most profoundly affected [3]; neuronal loss in the cerebral cortex, hippocampus, hypothalamus, substantia nigra, and in other brain structures has also been reported [4].

The mechanism by which the mutant huntingtin $(\mathrm{mHtt})$ causes HD is still poorly understood. Normal HTT has been shown to have multiple distinct functions in cells, including antiapoptotic activity [5-7], roles in vesicular transport [8$11]$, neuronal gene transcription regulation $[12,13]$, and control of synaptic transmission $[14,15]$. Consequently, the expression of long glutamine stretches either in the context of an N-terminal fragment or full-length HTT disrupts a wide variety of biological functions in cellular as well as animal models.

To date, many animal models have been developed that closely mimic HD symptoms or pathology. Rodents (mouse, rat) and nonhuman primates have been used most extensively to test HD gene therapy strategies. In general, HD animal models can be divided into chemically induced and genetic models. The chemically induced HD models include excitotoxic lesion models (glutamate-, kainic acid-, quinolinic acid- (QA-) induced) [16-19] and mitochondrial dysfunction models (3-nitroprionic acid (3-NP), malonate induced) [20-22]. Since HD is known to be a hereditary disorder, genetic models aim to mimic the molecular pathogenesis of HD more closely than that of chemical lesion models. The main types of genetic HD models are represented by transgenic (bearing the full-length or N-terminal fragment of $\mathrm{Htt}$ in their genome) [23, 24] and knock-in (expressing pathological length CAG repeat inserted into endogenous Htt gene) [25-27] animals.

The majority of therapeutics currently used to treat HD are designed to ameliorate the symptomatology of the condition, that is, psychiatric agents for the control of behavioural symptoms, motor sedatives, cognitive enhancers [28-30], 
and neuroprotective agents [31-37]. These drugs have limited benefits and do not address the disease progression. Meanwhile, gene therapy provides promising approaches in treating $\mathrm{HD}$, and sidesteps the need to understand how expression of the expanded CAG repeat in $\mathrm{Htt}$ causes the disease. These can be broadly classed into strategies involving (i) increasing expression levels of growth factors, (ii) decreasing levels of mutant HTT, and (iii) restoring cell metabolism and transcriptional balance.

\section{Neuroprotective and Neuroregenerative Approaches for HD}

Since the behavioural phenotypes in HD arise from a progressive loss of mainly striatal and cortical neurons, neuroprotection and neurorestoration are one of the major gene therapy approaches being developed. Early gene therapy strategies for HD focused on the delivery of neurotrophic factor genes as a direct means for protecting vulnerable striatal neurons against mutant HTT-mediated toxicity. Other alternatives have included delivery of molecules aimed at directing neurogenesis-the production of new adult neurons to replace neurons lost in the disease [38].

Neurotrophic factors prevent cell death in degenerative processes and enhance growth and function of neurons. Several neurotrophic factors have shown promise as therapeutic agents in cell lines and animal models. Nerve growth factor (NGF) was the first trophic factor evaluated in an excitotoxic rodent model of HD. However, the potent protection from degeneration with nearly two-thirds of the neurons in the QA-injured striatum rescued was found only when NGF was delivered into striatum by ex vivo modified cell grafts [39-42], but not when it was infused directly [43, 44]. The infusions provided neuroprotection to the cholinergic neuron population within the striatum while GABAergic neurons that are preferentially vulnerable in $\mathrm{HD}$ were not protected. Alternative growth factor candidates have also been evaluated and beneficial effects were found with delivery of neurturin (NTN) and glial cell line-derived neurotrophic factor (GDNF). Adenoassociated virus- (AAV-) delivered NTN reduced the extent of striatal neuronal cell death $(24 \%$ cell loss in the NTN-treated group versus $\approx 43 \%$ in control groups), and attenuated functional disability associated with striatal lesions produced by 3-NP infusion. Locomotor coordination as assessed by performance on the accelerating rotarod of animals expressing the NTN-producing transgene was similar to those of unlesioned animals [45]. Similarly, AAV-mediated delivery of GDNF in rat [46] and mouse [47] models of HD has been shown to provide structural and functional neuroprotection, promoting approximately $70 \%$ neuron survival as compared to untreated animals and improving behavioural performances at the platform and the hind limb clasping tests to a near normal level. Ex vivo delivery of GDNF also reduced neuronal death and maintained motor functions, although to a lesser extent. This was attributable to lower levels of transgene expression compared to those achieved by direct in vivo viral-mediated gene transfer, also, GDNF expression appeared limited to the area immediately surrounding the transplant core, and only $1 \%$ of total cells injected survived 3 months after transplantation [46]. Interestingly, intrastriatal lentivirus-mediated delivery of GDNF did not ameliorate neurological and behavioural impairments in the R6/2 transgenic mice model of HD [47]. This might be due to the incorrect timing of the treatment administration: GDNF overexpression might have been initiated too late in the course of the disease, when mutant huntingtin may already have triggered an irreversible pathogenetic process. Also, dependence of the neuroprotective effect on the particular mechanism of cell death in transgenic versus chemical HD models may be possible.

Encouraging results were obtained following ciliary neurotrophic factor (CNTF) infusion. Although lentiviralmediated overexpression of CNTF in the striatum of YAC72 mice, a genetic mouse model of $\mathrm{HD}$, reduced hyperactivity in 5 and 8 month old mice, no differences in rotarod performance or feet-clasping was found compared to controls [48]. In excitotoxic rodent and primate models, CNTF provided significant neuroprotection when delivered by means of genetically modified baby hamster kidney (BHK) cell grafts encapsulated in semipermeable membrane [49], osmotic minipump [50], lentiviral [51] and adenoviral [52] transfer represented by a 52-64\% reduction in lesion volumes and twice as big cell survival rate versus control groups. The experiments using the first technique showed either no difference or only a slight difference in neuron density between CNTF-treated and unlesioned brains, except for the lateral caudate where the decrease in density was $11 \%$ for NeuN-immunoreactive and $18 \%$ for calbindinimmunoreactive neurons. CNTF-expressing BHK cell grafts were also employed in a phase I clinical study involving a small number of HD patients. Whilst no side effects were observed over a period of 2 years, no clinical benefit was observed in these patients, most likely due to the low amounts of CNTF release produced by many of the capsules and low survival of the encapsulated cells [53].

Much research on HD has focused on brain-derived neurotrophic factor (BDNF), which is considered to be the main candidate for neuroprotective therapeutic strategies for HD. BDNF has been shown to modulate the onset and severity of motor and cognitive functions in HD mouse models [5456]. Mutant huntingtin reduces the transcriptional activity of the BDNF promoters, thus reducing the synthesis of BDNF protein in the cerebral cortex $[12,13]$, where approximately $95 \%$ of striatal BDNF is produced before being transported to its striatal targets via the corticostriatal afferents. It has also been reported that HD patients have lowered levels of BDNF in the cerebral cortex and striatum [57], and BDNF levels are also decreased in many mouse and cell models of the disease [58]. Adenoviral (Ad) vector-mediated delivery of BDNF reduced the size of QA-induced lesions in rats by one half, with $64 \%$ of medium spiny projection neurons surviving in Ad/BDNF-treated animals compared to $46 \%$ of those in controls [59]. AAV-mediated delivery of BDNF in similar excitotoxic rat model also provided neuroprotection to vulnerable striatal neurons $(71-78 \%$ versus $48-54 \%$ of preserved cells as shown by NeuN and calbindin immunoreactivity in BDNF-treated versus untreated striata, resp.) [60] and ameliorated motor dysfunction in tests designed to 
show hemispherical imbalances in brain function resultant of unilateral QA lesioning [61]. In contrast, neither ex vivo BDNF gene delivery $[41,62]$, nor direct BDNF protein infusion [50] has proved efficient in preventing the loss of striatal projection neurons following lesioning, presumably as the dosage of BDNF delivered may not have been sufficient to provide neuroprotection.

Although increasing BDNF expression by means of viral vectors has led to encouraging results, a number of issues still remain to be resolved, as excess expression of the BDNF transgene can have a deleterious effect on neuronal circuits and learning and memory [63], and some of the vectors are toxic per se and can cause tumour formation due to accidental insertional mutagenesis [64]. Furthermore, the questions of timing relative to intervention in the disease process, and anatomical location with respect to administration of the vector, must be addressed before applying the approach in patients, as transport of a transgene in axonal tracts could lead to unexpected side effects $[65,66]$.

\section{Therapeutic Strategies Targeting Mutant Huntingtin}

More recently, the development of effective gene silencing approaches using RNA interference technology has led to evaluation of strategies aimed at selectively reducing mutant $\mathrm{Htt}$ expression. This is an attractive approach to therapy as it sidesteps the requirement to understand the mechanism by which mutant $H t t$ causes the disease pathology. The therapeutic promise of this direct approach is underpinned by a pivotal study demonstrating that repression of $\mathrm{mHtt}$ expression in a conditional mouse model could reverse the pathological features of the disease including formation of neuronal inclusions and abnormal motor behaviour [67]. Thus decreasing abnormal $\mathrm{mHtt}$ load using this approach during the disease course might facilitate better protein clearance by affected neurons and allow neurons to normalise changes induced by $\mathrm{mHtt}$. Moreover, the ability to instigate treatments prior to onset of disease symptoms that is afforded by genetic screening for individuals that have inherited the HD mutation allows greater opportunity to make a significant impact on disease progression, when neuronal dysfunction might be prevalent but significant neurodegeneration and depletion of cortical and striatal neuron populations are yet to take place.

Modified antisense short nucleotide technology provides fascinating opportunities for development of nucleic acidbased therapeutics. Peptide nucleic acid (PNA) peptide conjugates and locked nucleic acid (LNA) oligomers were found to be potent and allele-selective inhibitors of mutant $\mathrm{Htt}$ expression in HD human cell lines. PNAs are DNA/RNA mimics with an uncharged amide backbone, that increases the affinity of PNA hybridization and facilitates recognition of RNA target, and LNA is an RNA analogue that contains a methylene bridge between the $2^{\prime}$-oxygen and $4^{\prime}$-carbon of the ribose, which reduces the conformational flexibility of the ribose and confers outstanding affinity to complementary hybridization. A comparison in the same model cell line revealed that inhibition by PNAs and LNAs that target CAG repeats is more effective than inhibition by an siRNA that targets a deletion polymorphism [68].

Early RNA interference-based approaches involved targeted knockdown of $\mathrm{mHtt}$ transcripts using species-specific short hairpin (shRNA) or short-interfering RNA (siRNA) in HD transgenic mouse models. Sequence differences between mouse host and human genes allowed allele-specific silencing of the pathogenic human $\mathrm{Htt}$ transcripts in the setting of preserved expression of endogenous $\mathrm{Htt}$. Intraventricular infusions of lipid-encapsulated [69] or intrastriatal injections of cholesterol-conjugated [70] siRNAs effectively silenced mutant huntingtin transcripts by $\approx 70 \%$ leading to a $56-66 \%$ reduction in HD protein levels. As a consequence, numbers and the size of intranuclear inclusions were decreased, and R6/2 transgenic mice and mice with the AAV-mediated $\mathrm{Htt}$ expression, respectively, showed improved performance in motor tests including beam walking and an accelerating rotarod.

One of the major hurdles for gene silencing is effective delivery of siRNA sequences to affected cells. The chronic nature of this disease suggests that continuous and long-term expression of siRNA will be required. This can be achieved by chronic infusion but whether lifelong infusion can be tolerated is unclear. An alternative approach is to use viral vectors to achieve long-term expression of shRNA molecules. AAV-mediated brain delivery of shRNA directed against human mutant $H t t$ decreased mutant huntingtin mRNA and protein levels by $51-78 \%$ and $28-50 \%$, respectively, reduced numbers of aberrant nuclear inclusions, and improved disease-associated behaviours such as the feet-clasping phenotype in HD mice and rotarod performance as well as spontaneous exploratory forepaw use in rats [71-73]. In the context of a lentiviral vector gene delivery system, shRNA targeted to the human $\mathrm{Htt}$ mRNA reduced $\mathrm{Htt}$ mRNA levels by more than $80 \%$ and almost completely prevented loss of dopamine and cAMP-regulated phosphoprotein-32 (DARPP-32) expression, and restored deficits in striatal glucose metabolism and mitochondrial complex II activity in a rat HD model. Expression of shRNA after appearance of HD pathology also produced a drastic reduction in the lesion size, associated with a partial clearance of HTT inclusions [74].

While the results of these proof-of-concept studies are encouraging, one challenge in extending this concept to human HD subjects is the potential requirement to develop approaches that selectively silence the mutant allele whilst leaving the normal allele intact. Normal huntingtin has roles in axonal guidance, cAMP signaling, long-term potentiation/ depression, and calcium and glutamate signaling, raising concerns about potential toxicity resulting from loss of huntingtin, function [58]. Moreover HD gene knockout in mice causes developmental defects and embryonic lethality [7577]. However, humans that are homozygote for the HD mutation $[78,79]$ and knock-in mice $[33,80,81]$ do exist, despite expressing no normal huntingtin, suggesting knockdown of normal $\mathrm{Htt}$ might be tolerated to some extent in the adult brain. To determine whether silencing of the normal allele might exacerbate mHtt pathology, Drouet et al. [74] and Boudreau et al. [82] evaluated the effect of knockdown sequences that would attenuate expression of both mutant 
and normal $H t t$ alleles. Lentiviral vector-mediated expression of a pathogenic human htt171-82Q on a background of reduced wild-type rat $\mathrm{Htt}$ levels did not produce any differences in GABAergic neuron survival or HTT inclusion load compared to rats that received the htt171-82Q alone suggesting partial inactivation of the endogenous rat allele did not increase the vulnerability of striatal neurons to $\mathrm{mHtt}$. Moreover, coincident nonallele-specific silencing of $\mathrm{mHtt}$ and wild-type $\mathrm{Htt}$ is well tolerated in mice, with minimal signs of toxicity for up to 9 months [74]. However in the context of an AAV vector system, shRNAs have been associated with toxicity, potentially due to saturation of cellular RNAi processing mechanisms $[82,83]$ which may limit usage of this technology. Interestingly, placing the identical siRNA sequences into artificial miRNA backbones mitigates the toxic effects markedly [82, 84]. Moreover, switching the RNA interference mechanism toward that used by microRNAs (miRNA) by introducing one or more mismatched bases into these duplex RNAs might allow preferential silencing of the mutant allele over the normal allele [85]. Transgenic HD171-82Q mice that received intrastriatal injections of an AAV vector expressing an miRNA sequence (mi2.4) that silences both mutant and wild-type mouse $\mathrm{Htt}$ mRNAs by $\approx 60 \%$ showed significant improvements in rotarod performance as compared to control-treated HD171-82Q mice. Moreover, despite AAV1-mi2.4 failing to normalise the weight loss observed in these animals, survival rates of these mice were also increased [82].

An alternative approach might also involve targeting disease-allele-linked small nucleotide polymorphisms (SNPs) [86] or deletion polymorphisms [87]. This provides the opportunity to develop reagents selectively silencing the disease-causing allele. Many SNPs are prevalent on the $\mathrm{mHtt}$ transcript [88], and it has been suggested that only 5 duplex siRNAs would be sufficient to treat $75 \%$ of patients with $\mathrm{HD}$, although the need to develop several reagents may be a complicating factor [89]. It is also unclear whether duplex RNAs will be able to achieve sufficient allele selectivity and potency in a therapeutic setting.

While these results are encouraging and suggest stringent targeted knockdown of the mutant allele may not be necessary, these studies have also reported significant transcriptomic changes in $\mathrm{Htt}$-related molecular pathways when expression of the wild-type huntingtin is inhibited [74, 82]. Boudreau et al. [82] compared datasets of transcripts differentially expressed by $>2$-fold following knockdown of normal $\mathrm{Htt}$ in mouse striatum with a dataset obtained from early grade HD patient caudate-putamen relative to their respective controls. Ninety-two genes were found to be altered in both gene sets. Interestingly, 41 of these showed changes in expression level in the same direction and were enriched in pathways involving developmental regulation of gene transcription. Fifty-one other genes that were common to both gene sets changed in the opposite direction and were enriched for proteins involved in ion transport and synaptic transmission, many of which are downregulated in human HD. These authors propose that these transcriptional changes might result from toxic gain of function aspect and that knocking down both mutant and wild-type Htt might revert these changes. However, a recent and extensive analysis of gene expression changes altered by Htt deficiency compared with the effect of the gain of function conferred by increasing CAG repeat length suggests that it is important to understand the biological processes affected by the lowering of normal $\mathrm{Htt}$ expression [90]. Panels of mouse embryonic stem (ES) cell lines engineered to express a full-length huntingtin mouse homolog (Hdh) with a knock-in of CAG repeats of increasing lengths were used to distinguish between gene expression changes conferred by the CAG expansion to that produced by loss of the endogenous $\mathrm{Htt}$ allele (Hdh null). Microarray comparison between a gene set whose expression was continuously altered with increasing CAG length with that of a huntingtin-null gene set showed that there was virtually no overlap in these gene sets suggesting the CAG expansion confers a simple gain of a novel function as opposed to a mixed gain of function/loss of function mechanism. Although the molecular responses are quite distinct, the gene expression changes result in varying degrees of interconnectedness at the network level. For example, pathways involved in the energy network such as carbohydrate metabolism/glycolysis are more prominently affected by CAG repeat length whereas oxidative respiration and tricarboxylic acid cycle pathways are more affected by huntingtin deficiency [90]. These authors suggest that treatments aimed at lowering the expression of the expanded CAG allele might instead exacerbate the physiologic effects of the expanded CAG repeat and thus gaining further insight into the biological processes affected by the lack of normal huntingtin is important. Thus selectively reducing expression of the disease-causing trigger without affecting the normal allele would be a preferable approach.

An alternative to knocking down $\mathrm{Htt}$ mRNA expression involves boosting the capacity of the cell to lower amounts of mutant huntingtin protein. The neurodegenerative and motor, cognitive, and psychiatric symptoms of HD typically manifest in midlife suggesting that in the presymptomatic phase, neurons are able to cope to some extent with the expression of mutant huntingtin protein. In a conditional transgenic mouse model of $\mathrm{HD}$, blockade of $\mathrm{mHtt}$ fragment expression in symptomatic mice leads to a disappearance of inclusions and an improvement in behavioural phenotypes, demonstrating that continuous production of the mutant protein is required to maintain the disease process and raising the possibility that HD may be reversible [67]. The half-life and clearance of normal and mutant HTT has not been studied in detail, however, enhancing the activity of molecular chaperones can promote refolding of misfolded proteins. For example, overexpression of one or both of the chaperones HSP104 and HSP27 has been shown to suppress mutant HTT-mediated neurotoxicity in a rat model of HD, promoting 59\% neuronal survival in vitro and reducing striatal lesion by 35-65\% in vivo [91]. Another therapeutic strategy uses intracellular antibodies (intrabodies) to target huntingtin. The intrabodies are recombinant antibodies maintaining the diversity, high specificity, and high affinity to the target site, characteristics of traditional antibodies, but they are smaller in size and have been genetically engineered to cross the blood-brain barrier and to function in 
the intracellular environment [92]. An intrabody approach has been assessed in various mouse models of HD [93]. It improved motor performance in the rotarod, beam crossing, climbing, and feet-clasping tests, and strongly ameliorated neuropathology including decreased numbers of striatal aggregates and a 2-2.5-fold reduction in lesion size in the intrabody-treated animals compared to untreated controls. In addition, G-rich oligonucleotide-based techniques that inhibit mutant HTT aggregation might also be of therapeutic value. A 20-mer, all G-oligonucleotide (HDG) capable of adopting a certain conformation, has been shown to block aggregation of a fusion protein that contained an $\mathrm{N}$-fragment of huntingtin bearing an aberrant polyglutamine tract [94].

\section{Strategies Promoting Cell Metabolism and Restoring Transcriptional Balance}

An important aspect of HD pathogenesis is the impact of the mutant protein on mitochondrial function and cellular bioenergetics. Reduced glucose utilisation and activities of complexes of the electron transporter chain in the striatum of advanced-grade HD subjects suggests a general metabolic deficit in HD patients [95-98]. Additionally, the sensitivity of medium spiny neurons to mitochondrial poisons such as 3-NP suggests the disease is influenced by altered mitochondrial function [99]. Mutant HTT has direct and/or indirect effects on mitochondria, compromising cellular energy production and respiration that leads to a reduction of the intracellular level of ATP, thus promoting apoptosis, oxidative stress and susceptibility to excitotoxicity $[100,101]$.

Therapeutic agents that aim to ameliorate the cellular energy deficits by enhancing energy production and improvement of mitochondrial function in HD are neuroprotective. Lentiviral-mediated overexpression of two subunits of the succinate dehydrogenase (SDH) enzyme, the main component of mitochondrial complex II, restores the membrane potential and blocks neuronal death induced by mutant huntingtin in murine cell model of HD [102]. Another key target of mutant huntingtin is peroxisome proliferatoractivated receptor gamma coactivator-1 $\alpha$ (PGC- $1 \alpha$ ), a transcriptional coactivator that regulates expression of genes involved in mitochondrial biogenesis and oxidative phosphorylation [103]. PGC- $1 \alpha$ expression levels are specifically reduced in the caudate-putamen, the first region affected in $\mathrm{HD}$, in presymptomatic postmortem HD cases. Moreover, a differential cellular expression pattern of PGC- $1 \alpha$ is found in the caudate-putamen, with decreased expression in the vulnerable medium spiny neurons but upregulation in nNOS-immunoreactive interneurons which are typically spared in the disease $[104,105]$. Decreased PGC- $1 \alpha$ expression is due to $\mathrm{mHtt}$-mediated repression of PGC- $1 \alpha$ transcription via interference of CREB/TAF4-dependent regulation of PGC- $1 \alpha$ gene expression. Inhibition of PGC1a expression leads to altered expression of genes involved in energy metabolism and diminished capacity of vulnerable neurons in response to energy demands in HD [105]. Cui et al. [105] determined whether genetic overexpression of PGC- $1 \alpha$ could protect against $\mathrm{mHtt}$-induced mitochondrial dysfunction and striatal toxicity. Direct administration of lentiviral vector expressing PGC- $1 \alpha$ into the striatum of R6/2 transgenic HD mice completely prevented neuronal atrophy, complementing in vitro data showing PGC- $1 \alpha$ overexpression reversed mitochondrial dysfunction in mutant STHdhQ111 cells, and abrogated the toxicity of $\mathrm{mHtt}$ in transfected primary striatal neurons, potentially through inducing the expression of genes encoding reactive oxygen species defence enzymes including $\mathrm{Cu} / \mathrm{Zn}$ superoxide dismutase (SOD1), manganese SOD (SOD2), catalase, and glutathione peroxidase $[105,106]$. This is in line with findings showing overexpression of SOD1, and molecular chaperones Hsp40 or Hsp70 in mutant HTT expressing mouse cells, prevents oxidative stress-induced proteasomal malfunction, mutant huntingtin aggregation, and cell death [107]. Whether PGC- $1 \alpha$ overexpression results in improved survival and motor performance in the R6/2 mice remains to be confirmed. These results suggest that stimulation of pathways involved in energy metabolism controlled by PGC- $1 \alpha$ by pharmacological or genetic means could provide potential clinical benefit at early stages of HD.

Huntingtin can interact with a number of transcription factors, for example, cAMP-response element binding (CREB) binding protein $[108,109]$, TATA-binding protein (TBP) [110], Sp1 [111], and p53 [109, 112], and consequently, transcriptional dysregulation occurs in the presence of mutant huntingtin [113]. One of the key genes, whose function is impaired in HD, is repressor element 1 silencing transcription factor (REST), a global repressor of neuronal gene expression, including BDNF. Disruption of REST target gene expression might be an early molecular event in HD [114] and thus attenuation of REST binding during early disease stages could be of therapeutic benefit. The employment of double-stranded oligodeoxynucleotide decoys corresponding to the DNA-binding element of the REST has been shown to abrogate its transcriptional activity and RESTmediated epigenetic repression rescuing levels of its target genes' mRNA and protein in the cell model of HD [115].

\section{Current Challenges in Development of Gene Therapy for HD}

Despite significant achievements in nucleic acid-based gene therapy for $\mathrm{HD}$, a range of technical problems is needed to be solved and questions to be answered. Each strategy described has specific disadvantages. Though many studies have proven that gene therapy approaches employing antisense oligonucleotides represent an exciting therapeutic possibility, there are certain issues that remain to be addressed. These include the choice of cellular targets, the stability of gene silencing, as well as side effects, such as altering off-target gene expression, induction of cellular immune response, and interfering with endogenous mRNA silencing systems. There are also problems associated with viral delivery of therapeutic genes. Transgene expression can be difficult to standardize and regulate, and some of the vectors can be toxic per se and/or cause insertional mutagenesis. The incorporation of a regulatory systems such as the tet system might increase the safety profile of such a treatment approach. Moreover, although studies using viral vectors have shown sustained gene 
expression for many years, maintaining long-term expression is still a current challenge.

Apart from these particular impediments, there are basic issues typical of all strategies, such as invasiveness of delivery methods associated with the direct administration of therapeutics into the brain due to the presence of blood-brain barrier, restriction of therapeutics distribution only to the area adjacent to the injection or transplantation site, and the need to ensure continuous effect of therapy and appropriate timing with relation to when to intervene in the course of disease.

Finally, the main obstacles remaining include our inability to distinguish between primary and secondary disease mechanisms. This raises the question as to which of the many cellular pathways of pathogenesis would be the most effective target in influencing disease onset and progression. Further identification of abnormalities, pathways, and targets that are the most critical for neurodegeneration and discriminating them from the ones that are secondary responses or just related phenomena are required.

\section{Acknowledgments}

This paper was supported by a University of Auckland International Doctoral Scholarship (T. Vagner) and funding from the Royal Society Marsden Fund and New Zealand Health Research Council (D. Young).

\section{References}

[1] J. B. Martin and J. F. Gusella, "Huntington's disease: pathogenesis and management," The New England Journal of Medicine, vol. 315, no. 20, pp. 1267-1276, 1986.

[2] "A novel gene containing a trinucleotide repeat that is expanded and unstable on Huntington's disease chromosomes. The Huntington's Disease Collaborative Research Group," Cell, vol. 72, no. 6, pp. 971-983, 1993.

[3] A. Reiner, R. L. Albin, K. D. Anderson, C. J. D'Amato, J. B. Penney, and A. B. Young, "Differential loss of striatal projection neurons in Huntington disease," Proceedings of the National Academy of Sciences of the United States of America, vol. 85, no. 15, pp. 5733-5737, 1988.

[4] J. P. G. Vonsattel and M. DiFiglia, "Huntington disease," Journal of Neuropathology and Experimental Neurology, vol. 57, no. 5, pp. 369-384, 1998.

[5] D. Rigamonti, J. H. Bauer, C. De-Fraja et al., "Wild-type huntingtin protects from apoptosis upstream of caspase-3," Journal of Neuroscience, vol. 20, no. 10, pp. 3705-3713, 2000.

[6] D. Rigamonti, S. Sipione, D. Goffredo, C. Zuccato, E. Fossale, and E. Cattaneo, "Huntingtin's neuroprotective activity occurs via inhibition of procaspase-9 processing," The Journal of Biological Chemistry, vol. 276, no. 18, pp. 14545-14548, 2001.

[7] B. R. Leavitt, J. M. Van Raamsdonk, J. Shehadeh et al., "Wild-type huntingtin protects neurons from excitotoxicity," Journal of Neurochemistry, vol. 96, no. 4, pp. 1121-1129, 2006.

[8] M. DiFiglia, E. Sapp, K. Chase et al., "Huntingtin is a cytoplasmic protein associated with vesicles in human and rat brain neurons," Neuron, vol. 14, no. 5, pp. 1075-1081, 1995.
[9] J. Block-Galarza, K. O. Chase, E. Sapp et al., "Fast transport and retrograde movement of huntingtin and HAP 1 in axons," NeuroReport, vol. 8, no. 9-10, pp. 2247-2251, 1997.

[10] L. R. Gauthier, B. C. Charrin, M. Borrell-Pagès et al., "Huntingtin controls neurotrophic support and survival of neurons by enhancing BDNF vesicular transport along microtubules," Cell, vol. 118, no. 1, pp. 127-138, 2004.

[11] E. Trushina, R. B. Dyer, J. D. Badger et al., "Mutant huntingtin impairs axonal trafficking in mammalian neurons in vivo and in vitro," Molecular and Cellular Biology, vol. 24, no. 18, pp. 8195-8209, 2004.

[12] C. Zuccato, A. Ciammola, D. Rigamonti et al., "Loss of huntingtin-mediated BDNF gene transcription in Huntington's disease," Science, vol. 293, no. 5529, pp. 493-498, 2001.

[13] C. Zuccato, M. Tartari, A. Crotti et al., "Huntingtin interacts with REST/NRSF to modulate the transcription of NRSEcontrolled neuronal genes," Nature Genetics, vol. 35, no. 1, pp. 76-83, 2003.

[14] Y. Sun, A. Savanenin, P. H. Reddy, and Y. F. Liu, "Polyglutamine-expanded huntingtin promotes sensitization of $\mathrm{N}$ methyl-D-aspartate receptors via post-synaptic density 95," The Journal of Biological Chemistry, vol. 276, no. 27, pp. 24713-24718, 2001.

[15] R. Smith, P. Brundin, and J. Y. Li, "Synaptic dysfunction in Huntington's disease: a new perspective," Cellular and Molecular Life Sciences, vol. 62, no. 17, pp. 1901-1912, 2005.

[16] E. G. McGeer and P. L. McGeer, "Duplication of biochemical changes of Huntington's chorea by intrastriatal injections of glutamic and kainic acids," Nature, vol. 263, no. 5577, pp. 517-519, 1976.

[17] M. F. Beal, P. E. Marshall, and G. D. Burd, "Excitotoxin lesions do not mimic the alteration of somatostatin in Huntington's disease," Brain Research, vol. 361, no. 1-2, pp. 135$145,1985$.

[18] M. F. Beal, N. W. Kowall, and D. W. Ellison, "Replication of the neurochemical characteristics of Huntington's disease by quinolinic acid," Nature, vol. 321, no. 6066, pp. 168-171, 1986.

[19] R. C. Roberts, A. Ahn, K. J. Swartz, M. F. Beal, and M. DiFiglia, "Intrastriatal injections of quinolinic acid or kainic acid: differential patterns of cell survival and the effects of data analysis on outcome," Experimental Neurology, vol. 124, no. 2, pp. 274-282, 1993.

[20] P. Hantraye, D. Riche, M. Maziere, and O. Isacson, "A primate model of Huntington's disease: behavioral and anatomical studies of unilateral excitotoxic lesions of the caudateputamen in the baboon," Experimental Neurology, vol. 108, no. 2, pp. 91-104, 1990.

[21] C. V. Borlongan, T. K. Koutouzis, T. B. Freeman, D. W. Cahill, and P. R. Sanberg, "Behavioral pathology induced by repeated systemic injections of 3-nitropropionic acid mimics the motoric symptoms of Huntington's disease," Brain Research, vol. 697, no. 1-2, pp. 254-257, 1995.

[22] M. C. Guyot, P. Hantraye, R. Dolan, S. Palfi, M. Maziére, and E. Brouillet, "Quantifiable bradykinesia, gait abnormalities and Huntington's disease- like striatal lesions in rats chronically treated with 3-nitropropionic acid," Neuroscience, vol. 79, no. 1, pp. 45-56, 1997.

[23] L. Mangiarini, K. Sathasivam, M. Seller et al., "Exon I of the HD gene with an expanded CAG repeat is sufficient to cause a progressive neurological phenotype in transgenic mice," Cell, vol. 87, no. 3, pp. 493-506, 1996.

[24] J. G. Hodgson, N. Agopyan, C. A. Gutekunst et al., "A YAC mouse model for Huntington's disease with full-length 
mutant huntingtin, cytoplasmic toxicity, and selective striatal neurodegeneration," Neuron, vol. 23, no. 1, pp. 181-192, 1999.

[25] P. F. Shelbourne, N. Killeen, R. F. Hevner et al., "A Huntington's disease CAG expansion at the murine Hdh locus is unstable and associated with behavioural abnormalities in mice," Human Molecular Genetics, vol. 8, no. 5, pp. 763-774, 1999.

[26] C. H. Lin, S. Tallaksen-Greene, W. M. Chien et al., "Neurological abnormalities in a knock-in mouse model of Huntington's disease," Human Molecular Genetics, vol. 10, no. 2, pp. 137-144, 2001.

[27] L. B. Menalled, J. D. Sison, Y. Wu et al., "Early motor dysfunction and striosomal distribution of huntingtin microaggregates in Huntington's disease knock-in mice," Journal of Neuroscience, vol. 22, no. 18, pp. 8266-8276, 2002.

[28] I. Reuter, M. T. M. Hu, T. C. Andrews, D. J. Brooks, C. Clough, and K. Ray Chaudhuri, "Late onset levodopa responsive Huntington's disease with minimal chorea masquerading as Parkinson plus syndrome," Journal of Neurology Neurosurgery and Psychiatry, vol. 68, no. 2, pp. 238-241, 2000.

[29] D. M. Blass, M. Steinberg, I. Leroi, and C. G. Lyketsos, "Successful multimodality treatment of severe behavioral disturbance in a patient with advanced Huntington's disease," American Journal of Psychiatry, vol. 158, no. 12, pp. 19661972, 2001.

[30] W. G. Ondo, R. Tintner, M. Thomas, and J. Jankovic, “Tetrabenazine treatment for Huntington's disease-associated chorea," Clinical Neuropharmacology, vol. 25, no. 6, pp. 300302, 2002.

[31] K. Kieburtz, A. Feigin, M. McDermott et al., "A controlled trial of remacemide hydrochloride in Huntington's disease," Movement Disorders, vol. 11, no. 3, pp. 273-277, 1996.

[32] B. Kremer, C. M. Clark, E. W. Almqvist et al., "Influence of lamotrigine on progression of early Huntington disease: a randomized clinical trial," Neurology, vol. 53, no. 5, pp. 10001011, 1999.

[33] K. Kieburtz, "A randomized, placebo-controlled trial of coenzyme Q10 and remacemide in Huntington's disease," Neurology, vol. 57, no. 3, pp. 397-404, 2001.

[34] F. J. Marshall, "Dosage effects of riluzole in Huntington's disease: a multicenter placebo-controlled study," Neurology, vol. 61, no. 11, pp. 1551-1556, 2003.

[35] S. J. Tabrizi, A. M. Blamire, D. N. Manners et al., "Creatine therapy for Huntington's disease: clinical and MRS findings in a 1-year pilot study," Neurology, vol. 61, no. 1, pp. 141-142, 2003.

[36] M. Thomas, T. Ashizawa, and J. Jankovic, "Minocycline in Huntington's disease: a pilot study," Movement Disorders, vol. 19, no. 6, pp. 692-695, 2004.

[37] B. K. Puri, B. R. Leavitt, M. R. Hayden et al., "Ethyl-EPA in Huntington disease: a double-blind, randomized, placebocontrolled trial," Neurology, vol. 65, no. 2, pp. 286-292, 2005.

[38] S. R. Cho, A. Benraiss, E. Chmielnicki, A. Samdani, A. Economides, and S. A. Goldman, "Induction of neostriatal neurogenesis slows disease progression in a transgenic murine model of Huntington disease," Journal of Clinical Investigation, vol. 117, no. 10, pp. 2889-2902, 2007.

[39] D. M. Frim, J. Simpson, T. A. Uhler et al., "Striatal degeneration induced by mitochondrial blockade is prevented by biologically delivered NGF," Journal of Neuroscience Research, vol. 35, no. 4, pp. 452-458, 1993.

[40] D. F. Emerich, J. P. Hammang, E. E. Baetge, and S. R. Winn, "Implantation of polymer-encapsulated human nerve growth factor-secreting fibroblasts attenuates the behavioral and neuropathological consequences of quinolinic acid injections into rodent striatum," Experimental Neurology, vol. 130, no. 1, pp. 141-150, 1994.

[41] A. Martínez-Serrano and A. Björklund, "Protection of the neostriatum against excitotoxic damage by neurotrophinproducing, genetically modified neural stem cells," Journal of Neuroscience, vol. 16, no. 15, pp. 4604-4616, 1996.

[42] J. H. Kordower, E. Y. Chen, C. Winkler et al., "Grafts of EGF-responsive neural stem cells derived from GFAP-hNGF transgenic mice: trophic and tropic effects in a rodent model of Huntington's disease," Journal of Comparative Neurology, vol. 387, no. 1, pp. 96-113, 1997.

[43] J. H. Kordower, V. Charles, R. Bayer et al., "Intravenous administration of a transferrin receptor antibody-nerve growth factor conjugate prevents the degeneration of cholinergic striatal neurons in a model of Huntington disease," Proceedings of the National Academy of Sciences of the United States of America, vol. 91, no. 19, pp. 9077-9080, 1994.

[44] J. L. Venero, K. D. Beck, and F. Hefti, "Intrastriatal infusion of nerve growth factor after quinolinic acid prevents reduction of cellular expression of choline acetyltransferase messenger RNA and trkA messenger RNA, but not glutamate decarboxylase messenger RNA," Neuroscience, vol. 61, no. 2, pp. 257-268, 1994.

[45] S. Ramaswamy, J. L. McBride, C. D. Herzog et al., "Neurturin gene therapy improves motor function and prevents death of striatal neurons in a 3-nitropropionic acid rat model of Huntington's disease," Neurobiology of Disease, vol. 26, no. 2, pp. 375-384, 2007.

[46] A. D. Ebert, A. E. Barber, B. M. Heins, and C. N. Svendsen, "Ex vivo delivery of GDNF maintains motor function and prevents neuronal loss in a transgenic mouse model of Huntington's disease," Experimental Neurology, vol. 224, no. 1, pp. 155-162, 2010

[47] N. Popovic, M. Maingay, D. Kirik, and P. Brundin, "Lentiviral gene delivery of GDNF into the striatum of R6/2 Huntington mice fails to attenuate behavioral and neuropathological changes," Experimental Neurology, vol. 193, no. 1, pp. 65-74, 2005.

[48] D. Zala, J. C. Bensadoun, L. P. De Almeida et al., "Long-term lentiviral-mediated expression of ciliary neurotrophic factor in the striatum of Huntington's disease transgenic mice," Experimental Neurology, vol. 185, no. 1, pp. 26-35, 2004.

[49] V. Mittoux, J. M. Joseph, F. Conde et al., "Restoration of cognitive and motor functions by ciliary neurotrophic factor in a primate model of Huntington's disease," Human Gene Therapy, vol. 11, no. 8, pp. 1177-1187, 2000.

[50] K. D. Anderson, N. Panayotatos, T. L. Corcoran, R. M. Lindsay, and S. J. Wiegand, "Ciliary neurotrophic factor protects striatal output neurons in an animal model of Huntington disease," Proceedings of the National Academy of Sciences of the United States of America, vol. 93, no. 14, pp. 7346-7351, 1996.

[51] L. P. de Almeida, D. Zala, P. Aebischer, and N. Déglon, "Neuroprotective effect of a CNTF-expressing lentiviral vector in the quinolinic acid rat model of Huntington's disease," Neurobiology of Disease, vol. 8, no. 3, pp. 433-446, 2001.

[52] V. Mittoux, S. Ouary, C. Monville et al., "Corticostriatopallidal neuroprotection by adenovirus-mediated ciliary neurotrophic factor gene transfer in a rat model of progressive striatal degeneration," Journal of Neuroscience, vol. 22, no. 11, pp. 4478-4486, 2002. 
[53] J. Bloch, A. C. Bachoud-Lévi, N. Déglon et al., "Neuroprotective gene therapy for Huntington's disease, using polymerencapsulated cells engineered to secrete human ciliary neurotrophic factor: results of a phase I study," Human Gene Therapy, vol. 15, no. 10, pp. 968-975, 2004.

[54] G. Lynch, E. A. Kramar, C. S. Rex et al., "Brain-derived neurotrophic factor restores synaptic plasticity in a knock-in mouse model of Huntington's disease," Journal of Neuroscience, vol. 27, no. 16, pp. 4424-4434, 2007.

[55] J. M. Canals, J. R. Pineda, J. F. Torres-Peraza et al., "Brainderived neurotrophic factor regulates the onset and severity of motor dysfunction associated with enkephalinergic neuronal degeneration in Huntington's disease," Journal of Neuroscience, vol. 24, no. 35, pp. 7727-7739, 2004.

[56] A. Giralt, T. Rodrigo, E. D. Martín et al., "Brain-derived neurotrophic factor modulates the severity of cognitive alterations induced by mutant huntingtin: involvement of phospholipaseC $\gamma$ activity and glutamate receptor expression," Neuroscience, vol. 158, no. 4, pp. 1234-1250, 2009.

[57] I. Ferrer, E. Goutan, C. Marín, M. J. Rey, and T. Ribalta, "Brain-derived neurotrophic factor in Huntington disease," Brain Research, vol. 866, no. 1-2, pp. 257-261, 2000.

[58] E. Cattaneo, C. Zuccato, and M. Tartari, "Normal huntingtin function: an alternative approach to Huntington's disease," Nature Reviews Neuroscience, vol. 6, no. 12, pp. 919-930, 2005.

[59] A. P. Bemelmans, P. Horellou, L. Pradier, I. Brunet, P. Colin, and J. Mallet, "Brain-derived neurotrophic factor-mediated protection of striatal neurons in an excitotoxic rat model of Huntington's disease, as demonstrated by adenoviral gene transfer," Human Gene Therapy, vol. 10, no. 18, pp. 29872997, 1999.

[60] A. P. Kells, D. M. Fong, M. Dragunow, M. J. During, D. Young, and B. Connor, "AAV-mediated gene delivery of BDNF or GDNF is neuroprotective in a model of Huntington disease," Molecular Therapy, vol. 9, no. 5, pp. 682-688, 2004.

[61] A. P. Kells, R. A. Henry, and B. Connor, "AAV-BDNF mediated attenuation of quinolinic acid-induced neuropathology and motor function impairment," Gene Therapy, vol. 15, no. 13, pp. 966-977, 2008.

[62] D. M. Frim, T. A. Uhler, M. P. Short et al., "Effects of biologically delivered NGF, BDNF and bFGF on striatal excitotoxic lesions," NeuroReport, vol. 4, no. 4, pp. 367-370, 1993.

[63] S. Arancibia, M. Silhol, F. Moulière et al., "Protective effect of BDNF against beta-amyloid induced neurotoxicity in vitro and in vivo in rats," Neurobiology of Disease, vol. 31, no. 3, pp. 316-326, 2008.

[64] U. Modlich and C. Baum, "Preventing and exploiting the oncogenic potential of integrating gene vectors," The Journal of Clinical Investigation, vol. 119, no. 4, pp. 755-758, 2009.

[65] R. J. Mandel, F. P. Manfredsson, K. D. Foust et al., "Recombinant adeno-associated viral vectors as therapeutic agents to treat neurological disorders," Molecular Therapy, vol. 13, no. 3, pp. 463-483, 2006.

[66] F. P. Manfredsson and R. J. Mandel, "Development of gene therapy for neurological disorders," Discovery Medicine, vol. 9, no. 46, pp. 204-211, 2010.

[67] A. Yamamoto, J. J. Lucas, and R. Hen, "Reversal of neuropathology and motor dysfunction in a conditional model of Huntington's disease," Cell, vol. 101, no. 1, pp. 57-66, 2000.

[68] J. Hu, M. Matsui, and D. R. Corey, "Allele-selective inhibition of mutant huntingtin by peptide nucleic acid-peptide conjugates, locked nucleic acid, and small interfering RNA," Annals of the New York Academy of Sciences, vol. 1175, pp. 24-31, 2009.
[69] Y. L. Wang, W. Liu, E. Wada, M. Murata, K. Wada, and I. Kanazawa, "Clinico-pathological rescue of a model mouse of Huntington's disease by siRNA," Neuroscience Research, vol. 53, no. 3, pp. 241-249, 2005.

[70] M. DiFiglia, M. Sena-Esteves, K. Chase et al., "Therapeutic silencing of mutant huntingtin with siRNA attenuates striatal and cortical neuropathology and behavioral deficits," Proceedings of the National Academy of Sciences of the United States of America, vol. 104, no. 43, pp. 17204-17209, 2007.

[71] N. R. Franich, H. L. Fitzsimons, D. M. Fong, M. Klugmann, M. J. During, and D. Young, "AAV vector-mediated RNAi of mutant Huntingtin expression is neuroprotective in a novel genetic rat model of Huntington's disease," Molecular Therapy, vol. 16, no. 5, pp. 947-956, 2008.

[72] S. Q. Harper, P. D. Staber, X. He et al., "RNA interference improves motor and neuropathological abnormalities in a Huntington's disease mouse model," Proceedings of the National Academy of Sciences of the United States of America, vol. 102, no. 16, pp. 5820-5825, 2005.

[73] E. Rodriguez-Lebron, E. M. Denovan-Wright, K. Nash, A. S. Lewin, and R. J. Mandel, "Intrastriatal rAAV-mediated delivery of anti-huntingtin shRNAs induces partial reversal of disease progression in R6/1 Huntington's disease transgenic mice," Molecular Therapy, vol. 12, no. 4, pp. 618-633, 2005.

[74] V. Drouet, V. Perrin, R. Hassig et al., "Sustained effects of nonallele-specific huntingtin silencing," Annals of Neurology, vol. 65, no. 3, pp. 276-285, 2009.

[75] J. Nasir, S. B. Floresco, J. R. O'Kusky et al., "Targeted disruption of the Huntington's disease gene results in embryonic lethality and behavioral and morphological changes in heterozygotes," Cell, vol. 81, no. 5, pp. 811-823, 1995.

[76] W. Auerbach, M. S. Hurlbert, P. Hilditch-Maguire et al., "The HD mutation causes progressive lethal neurological disease in mice expressing reduced levels of huntingtin," Human Molecular Genetics, vol. 10, no. 22, pp. 2515-2523, 2001.

[77] J. K. White, W. Auerbach, M. P. Duyao et al., "Huntington is required for neurogenesis and is not impaired by the Huntington's disease CAG expansion," Nature Genetics, vol. 17, no. 4, pp. 404-410, 1997.

[78] N. S. Wexler, A. B. Young, and R. E. Tanzi, "Homozygotes for Huntington's disease," Nature, vol. 326, no. 6109, pp. 194197, 1987.

[79] R. H. Myers, J. Leavitt, L. A. Farrer et al., "Homozygote for Huntington disease," The American Journal of Human Genetics, vol. 45, no. 4, pp. 615-618, 1989.

[80] V. C. Wheeler, C. A. Gutekunst, V. Vrbanac et al., "Early phenotypes that presage late-onset neurodegenerative disease allow testing of modifiers in Hdh CAG knock-in mice," Human Molecular Genetics, vol. 11, no. 6, pp. 633-640, 2002.

[81] V. C. Wheeler, J. K. White, C. A. Gutekunst et al., "Long glutamine tracts cause nuclear localization of a novel form of huntingtin in medium spiny striatal neurons in $\mathrm{Hdh}(\mathrm{Q} 92)$ and Hdh(Q111) knock-in mice," Human Molecular Genetics, vol. 9, no. 4, pp. 503-513, 2000.

[82] R. L. Boudreau, J. L. McBride, I. Martins et al., "Nonallelespecific silencing of mutant and wild-type huntingtin demonstrates therapeutic efficacy in Huntington's disease mice," Molecular Therapy, vol. 17, no. 6, pp. 1053-1063, 2009.

[83] R. L. Boudreau, I. Martins, and B. L. Davidson, "Artificial MicroRNAs as siRNA shuttles: improved safety as compared to shRNAs in vitro and In vivo," Molecular Therapy, vol. 17, no. 1, pp. 169-175, 2009.

[84] J. L. McBride, R. L. Boudreau, S. Q. Harper et al., "Artificial miRNAs mitigate shRNA-mediated toxicity in the brain: 
implications for the therapeutic development of RNAi," Proceedings of the National Academy of Sciences of the United States of America, vol. 105, no. 15, pp. 5868-5873, 2008.

[85] J. Hu, J. Liu, and D. R. Corey, "Allele-selective inhibition of huntingtin expression by switching to an miRNA-like RNAi mechanism," Chemistry and Biology, vol. 17, no. 11, pp. 1183-1188, 2010.

[86] E. L. Pfister, L. Kennington, J. Straubhaar et al., "Five siRNAs targeting three SNPs may provide therapy for three-quarters of Huntington's disease patients," Current Biology, vol. 19, no. 9, pp. 774-778, 2009.

[87] Y. Zhang, J. Engelman, and R. M. Friedlander, "Allele-specific silencing of mutant Huntington's disease gene," Journal of Neurochemistry, vol. 108, no. 1, pp. 82-90, 2009.

[88] W. Liu, L. A. Kennington, H. D. Rosas et al., "Linking SNPs to CAG repeat length in Huntington's disease patients," Nature Methods, vol. 5, no. 11, pp. 951-953, 2008.

[89] D. S. Schwarz, H. Ding, L. Kennington et al., "Designing siRNA that distinguish between genes that differ by a single nucleotide," PLoS Genetics, vol. 2, no. 9, pp. 1307-1318, 2006.

[90] J. C. Jacobsen, G. C. Gregory, J. M. Woda et al., "HD CAGcorrelated gene expression changes support a simple dominant gain of function," Human Molecular Genetics, vol. 20, no. 14, pp. 2846-2860, 2011.

[91] V. Perrin, E. Régulier, T. Abbas-Terki et al., "Neuroprotection by Hsp104 and Hsp27 in lentiviral-based rat models of Huntington's disease," Molecular Therapy, vol. 15, no. 5, pp. 903-911, 2007.

[92] A. Messer, S. M. Lynch, and D. C. Butler, "Developing intrabodies for the therapeutic suppression of neurodegenerative pathology," Expert Opinion on Biological Therapy, vol. 9, no. 9, pp. 1189-1197, 2009.

[93] A. L. Southwell, J. Ko, and P. H. Patterson, "Intrabody gene therapy ameliorates motor, cognitive, and neuropathological symptoms in multiple mouse models of Huntington's disease," Journal of Neuroscience, vol. 29, no. 43, pp. 13589 13602, 2009.

[94] M. Skogen, J. Roth, S. Yerkes, H. Parekh-Olmedo, and E. Kmiec, "Short G-rich oligonucleotides as a potential therapeutic for Huntington's Disease," BMC Neuroscience, vol. 7, article 65, 2006.

[95] S. T. Grafton, J. C. Mazziotta, J. J. Pahl et al., "Serial changes of cerebral glucose metabolism and caudate size in persons at risk for Huntington's disease," Archives of Neurology, vol. 49, no. 11, pp. 1161-1167, 1992.

[96] A. Antonini, K. L. Leenders, R. Spiegel et al., "Striatal glucose metabolism and dopamine D2 receptor binding in asymptomatic gene carriers and patients with Huntington's disease," Brain, vol. 119, part 6, pp. 2085-2095, 1996.

[97] T. Kuwert, H. W. Lange, H. Boecker et al., "Striatal glucose consumption in chorea-free subjects at risk of Huntington's disease," Journal of Neurology, vol. 241, no. 1, pp. 31-36, 1993.

[98] S. E. Browne and M. F. Beal, "The energetics of Huntington's disease," Neurochemical Research, vol. 29, no. 3, pp. 531-546, 2004.

[99] M. F. Beal, E. Brouillet, B. G. Jenkins et al., "Neurochemical and histologic characterization of striatal excitotoxic lesions produced by the mitochondrial toxin 3-nitropropionic acid," Journal of Neuroscience, vol. 13, no. 10, pp. 4181-4192, 1993.

[100] S. E. Browne, "Mitochondria and Huntington's disease pathogenesis: insight from genetic and chemical models," Annals of the New York Academy of Sciences, vol. 1147, pp. 358-382, 2008.
[101] S. Imarisio, J. Carmichael, V. Korolchuk et al., "Huntington's disease: from pathology and genetics to potential therapies," Biochemical Journal, vol. 412, no. 2, pp. 191-209, 2008.

[102] A. Benchoua, Y. Trioulier, D. Zala et al., "Involvement of mitochondrial complex II defects in neuronal death produced by N-terminus fragment of mutated huntingtin," Molecular Biology of the Cell, vol. 17, no. 4, pp. 1652-1663, 2006.

[103] P. Puigserver and B. M. Spiegelman, "Peroxisome proliferator-activated receptor- $\gamma$ coactivator $1 \alpha(\mathrm{PGC}-1 \alpha)$ : transcriptional coactivator and metabolic regulator," Endocrine Reviews, vol. 24, no. 1, pp. 78-90, 2003.

[104] R. J. Ferrante, N. W. Kowall, and M. F. Beal, "Selective sparing of a class of striatal neurons in Huntington's disease," Science, vol. 230, no. 4725, pp. 561-563, 1985.

[105] L. Cui, H. Jeong, F. Borovecki, C. N. Parkhurst, N. Tanese, and D. Krainc, "Transcriptional repression of PGC- $1 \alpha$ by mutant huntingtin leads to mitochondrial dysfunction and neurodegeneration," Cell, vol. 127, no. 1, pp. 59-69, 2006.

[106] J. St-Pierre, S. Drori, M. Uldry et al., "Suppression of Reactive Oxygen Species and Neurodegeneration by the PGC-1 Transcriptional Coactivators," Cell, vol. 127, no. 2, pp. 397-408, 2006.

[107] A. Goswami, P. Dikshit, A. Mishra, S. Mulherkar, N. Nukina, and N. R. Jana, "Oxidative stress promotes mutant huntingtin aggregation and mutant huntingtin-dependent cell death by mimicking proteasomal malfunction," Biochemical and Biophysical Research Communications, vol. 342, no. 1, pp. 184-190, 2006.

[108] F. C. Nucifora, M. Sasaki, M. F. Peters et al., "Interference by huntingtin and atrophin-1 with CBP-mediated transcription leading to cellular toxicity," Science, vol. 291, no. 5512, pp. 2423-2428, 2001.

[109] J. S. Steffan, A. Kazantsev, O. Spasic-Boskovic et al., "The Huntington's disease protein interacts with p53 and CREBbinding protein and represses transcription," Proceedings of the National Academy of Sciences of the United States of America, vol. 97, no. 12, pp. 6763-6768, 2000.

[110] C. C. Huang, P. W. Faber, F. Persichetti et al., "Amyloid formation by mutant huntingtin: threshold, progressivity and recruitment of normal polyglutamine proteins," Somatic Cell and Molecular Genetics, vol. 24, no. 4, pp. 217-233, 1998.

[111] A. W. Dunah, H. Jeong, A. Griffin et al., "Sp1 and TAFII130 transcriptional activity disrupted in early Huntington's disease," Science, vol. 296, no. 5576, pp. 2238-2243, 2002.

[112] B. I. Bae, H. Xu, S. Igarashi et al., "p53 mediates cellular dysfunction and behavioral abnormalities in Huntington's disease," Neuron, vol. 47, no. 1, pp. 29-41, 2005.

[113] D. C. Rubinsztein and J. Carmichael, "Huntington's disease: molecular basis of neurodegeneration," Expert Reviews in Molecular Medicine, vol. 5, no. 20, pp. 1-21, 2003.

[114] C. Zuccato, N. Belyaev, P. Conforti et al., "Widespread disruption of repressor element-1 silencing transcription factor/ neuron-restrictive silencer factor occupancy at its target genes in Huntington's disease," Journal of Neuroscience, vol. 27, no. 26, pp. 6972-6983, 2007.

[115] C. Soldati, A. Bithell, P. Conforti, E. Cattaneo, and N. J. Buckley, "Rescue of gene expression by modified REST decoy oligonucleotides in a cellular model of Huntington's disease," Journal of Neurochemistry, vol. 116, no. 3, pp. 415-425, 2011. 


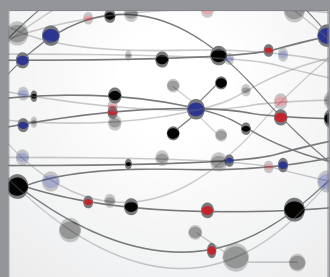

The Scientific World Journal
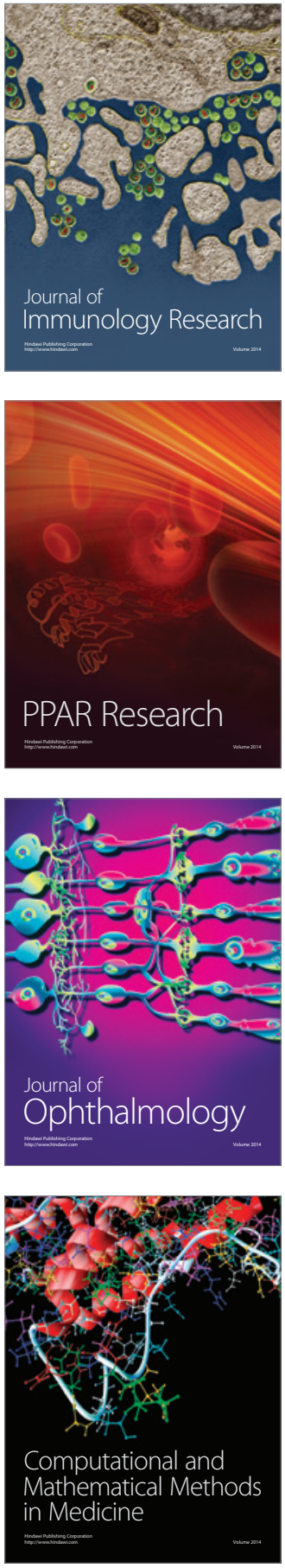

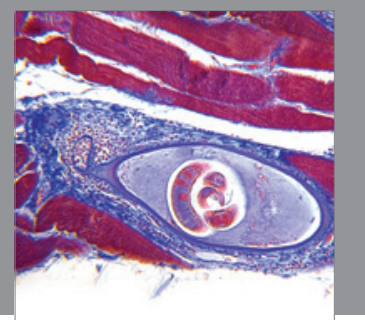

Gastroenterology

Research and Practice
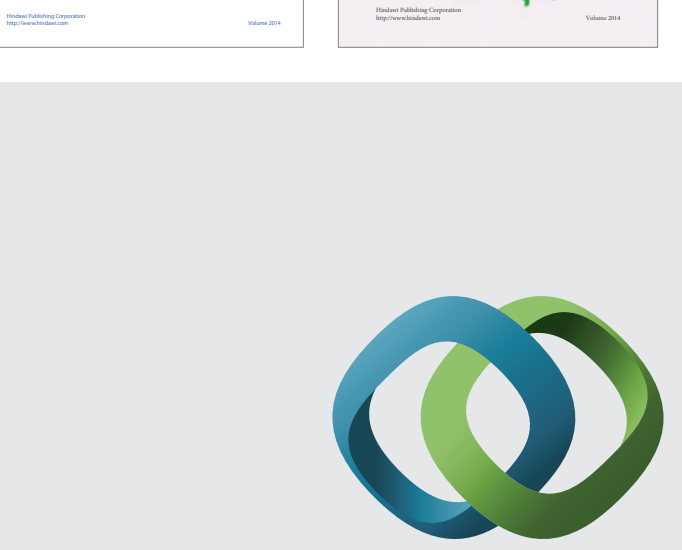

\section{Hindawi}

Submit your manuscripts at

http://www.hindawi.com
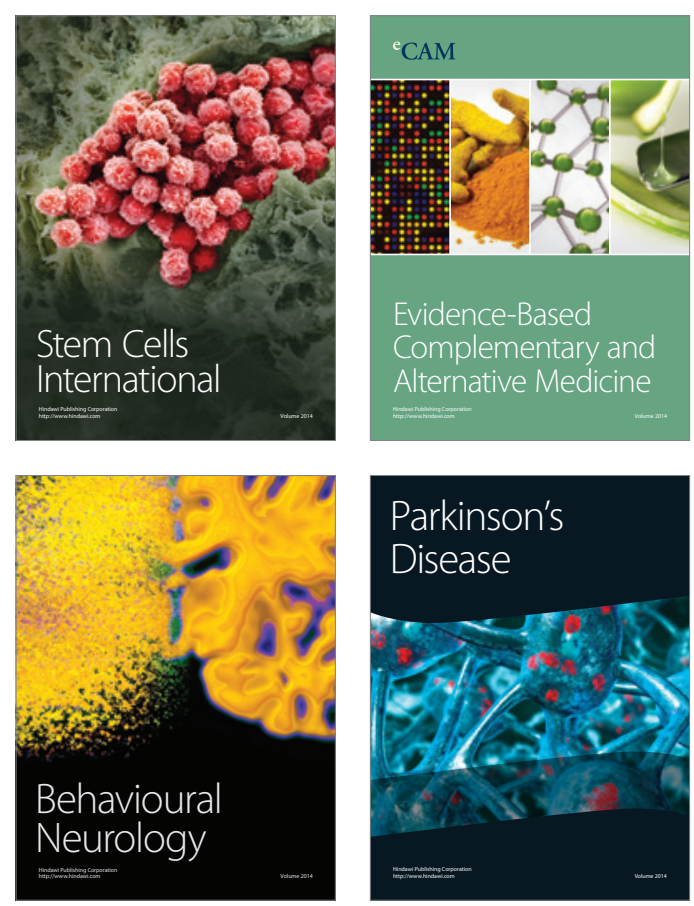

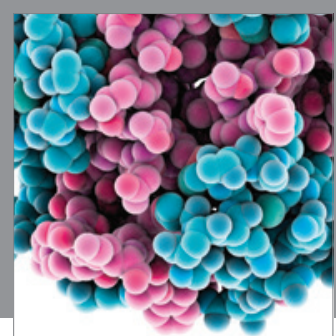

Journal of
Diabetes Research

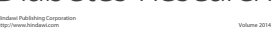

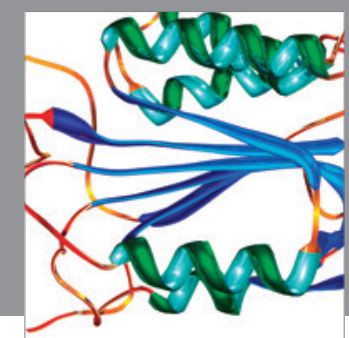

Disease Markers
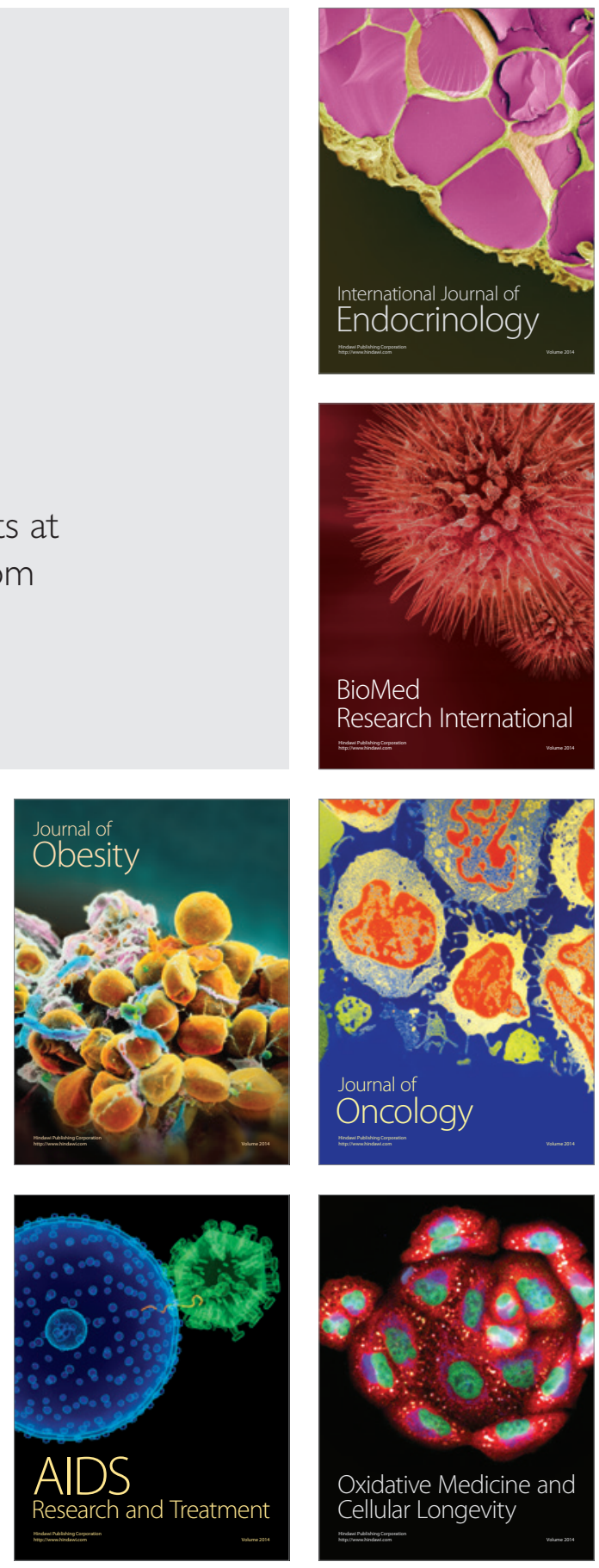